\title{
Clinical symptom dimensions and deficits on the Continuous Performance Test in schizophrenia
}

\author{
Shi K. Liu a , Hai-Gwo Hwu a , Wei J. Chen ${ }^{\text {b.* }}$ \\ a Department of Psychiatry, College of Medicine, National Taiwan University, Taipei, Taiwan \\ ${ }^{\mathrm{b}}$ Institute of Epidemiology, College of Public Health, National Taiwan University. I Jen-Ai Road Sec. 1, Taipei 100, \\ Taiwan
}

Received 1 April 1996; accepted 4 March 1997

\begin{abstract}
We examined the relationships between symptom dimensions derived from factor analytic studies of schizophrenic symptoms and sustained attention deficits. Four factors, negative, delusion/hallucination, disorganization, and excitement, were yielded from factor analysis on 14 items of the Positive and Negative Syndrome Scale (PANSS) among 60 Chinese inpatients with acute schizophrenia. The negative dimension was associated with lower sensitivity index $\left(d^{\prime}\right)$ while the excitement dimension was associated with higher $d^{\prime}$ on the Continuous Performance Test (CPT) after sex, age and education were adjusted for in multiple linear regressions. The positive dimension affected only response criterion $(\ln \beta)$ and was not associated with the $d^{\prime}$ on the CPT. In contrast, the summed scores of PANSS Positive and Negative scales did not have significant correlations with $d^{\prime}$ on the CPT. Thus, the discriminant validity of these symptom dimensions of schizophrenia is supported by their correlations with CPT performance indices. (C) 1997 Elsevier Science B.V.
\end{abstract}

Keywords: Continuous Performance Test; Factor analysis; Positive and Negative Syndrome Scale; Schizophrenia; Symptom dimensions

\section{Introduction}

The positive/negative dichotomy of clinical symptoms of schizophrenia has a long history in phenomenological psychiatry (Berrios, 1991) and has led to both a two-syndrome model (Crow, 1980) and a unique dimension with a bipolar character model (Andreasen and Olsen, 1982). However, subsequent efforts have failed to demonstrate the validity of these models (Arndt et al.,

* Corresponding author. Tel.: + 886-2-3970800, ext. 8360; fax: $+886-2-3560840$.
1991; Carpenter et al., 1991; De Leon et al., 1992). Results of recent factor analytic studies of clinical symptoms suggested that more than two symptom dimensions are needed to represent the full spectrum of symptoms of schizophrenia (Liddle, 1987; Arndt et al., 1991; Andreasen et al., 1995; Lindenmayer et al., 1995; Lenzenweger and Dworkin, 1996; Johnstone and Frith, 1996; Vázquez-Barquero et al., 1996). However, the variations in the reported factor structures render their validity inconclusive. Differences in patient characteristics and symptom ensembles assessed might partly account for the discrepancies. One impor- 
tant step in further validating the factor structures and generating a heuristic model for the clinical symptoms is to explore their relationships with concomitant laboratory measures of putative pathological processes (Neale et al., 1985).

Sustained attention deficits detected by the Continuous Performance Test (CPT) (Rosvold et al., 1956) are one such potential validator. Schizophrenic patients in various clinical and medication states have been found to have deficits on CPT performance (Orzack and Kornetsky, 1966; Wohlberg and Kornetsky, 1973; Asarnow and MacCrimmon, 1978). The deficits are relatively specific for schizophrenia as compared to other psychiatric disorders (Cornblatt et al., 1989; Mussgay and Hertwig, 1990). Similar deficits on the CPT have also been demonstrated in the population at increased risk for schizophrenia (Rutschmann et al., 1977; Cornblatt et al., 1992). In addition, past studies specifically examining the relationships between CPT performance indices and clinical symptoms have shown, rather consistently, that CPT deficits were associated with specific dimensions of schizophrenic symptoms, especially negative symptoms (Nuechterlein et al., 1986; Hain et al., 1993; Johnstone and Frith, 1996) and thought disorders (Nuechterlein et al., 1986; Strauss et al., 1993; Pandurangi et al., 1994). However, these studies focused either on the global severity of hypothetical syndromes or symptom dimensions derived a priori from reported factor structures. The relationship between CPT deficit and empirically derived dimensions of schizophrenic symptoms remains to be examined. In addition, the influence of demographic features on the CPT performance was not adequately controlled for in previous studies. A large community study has shown that CPT performance is associated with age, sex and education (Chen et al., 1997a).

In this study, we set out to test the hypotheses that: (1) symptoms of schizophrenia consist of distinct symptom dimensions, rather than the dichotomized positive and negative syndromes; and (2) sustained attention deficits detected by the CPT are associated with specific symptom dimensions, especially the negative symptom dimension, rather than the global severity of hypothetical positive and negative syndromes, after controlling for demographic features of patients.

\section{Method}

\subsection{Subjects}

The subjects were derived from an ongoing prospective follow-up study for the validation of the negative/non-negative subtyping of schizophrenia, the Multidimensional Psychopathology Group Research Projects (MPGRP). Since August 1, 1993, patients consecutively admitted to the acute inpatient wards of three hospitals, the National Taiwan University Hospital, the Taipei City Psychiatric Center and the Provincial TaoYuan Psychiatric Center, were included in MPGRP if they met the Diagnostic and Statistical Manual of Mental Disorders, Third EditionRevised (DSM-III-R) (American Psychiatric Association, 1987) criteria for schizophrenic disorders and agreed to participate. The diagnoses were re-evaluated at discharge by consensus achieved by three senior psychiatrists using all information available from clinical observations, past medical records and key informants. All patients with a discharge diagnosis other than schizophrenia and patients with previous history of physical illness or substance abuse, which cast the diagnoses in doubt, were excluded.

As of December 31, 1995, 98 schizophrenic patients had been recruited for the MPGRP. Among them, 38 patients were not included in this study because of refusal to take the CPT $(n=28)$, being unable to complete two sessions of the CPT $(n=3)$, or a delay of more than 1 week between clinical symptom assessment and CPT $(n=7)$. There was no difference in sex, age or severity of psychopathology between those included in this study and those not included. Hence, the following analyses were based on a sample of 60 schizophrenic patients.

\subsection{Symptom-rating instrument}

The clinical symptoms of the patients were assessed by the Positive and Negative Syndrome 
Scale (PANSS) (Kay et al., 1987). The PANSS has guidelines for a semi-structured interview with detailed descriptions for symptom ratings, and has been thoroughly studied psychometrically (Kay, 1991). The weightings of positive and negative syndromes are assumed to be balanced in the PANSS, which is reflected in that the Positive scale and Negative scale each cover seven symptoms considered to comprise the core of individual syndromes (Kay, 1991). The severity of both syndromes are represented by the summed scores of the Positive and Negative scales, each ranging from 7 to 49 . The time period covered by each evaluation is 1 week, prior to the date of clinical evaluation.

A Chinese version of the PANSS, the PANSS-CH, was translated from the English version specifically for the MPGRP. The development of the PANSS-CH has been described in detail elsewhere (Cheng et al., 1996). In a subsequent reliability test of another 30 schizophrenic patients, good inter-rater reliabilities were achieved on the PANSS-CH. The ranges of intraclass correlation coefficient reliabilities (ICCRs) of the Positive scale items were $0.64-0.96$, and those of the Negative scale items were $0.64-0.95$.

\section{3. $C P T$}

We used a CPT machine from Sunrise Systems, v. 2.20 (Pembroke, MA, USA). The procedure has been described in detail elsewhere (Chen et al., 1997a). Briefly, numbers between 0 and 9 were randomly presented for $50 \mathrm{~ms}$ each, at a rate of $1 / \mathrm{s}$. Available response time was $700 \mathrm{~ms}$ and the probability of critical stimulus was set at $10 \%$. Each subject undertook two CPT sessions: the undegraded 1-9 task and the degraded 1-9 task. During the undegraded session, subjects responded to the target stimulus (the number 9 preceded by the number 1) by pressing a button. A total of 331 trials, 31 of them targets, were presented over 5 min for each session. During the degraded session, a pattern of snow was used to toggle background and foreground dots so that the image was not distinct. Two signal-detection indices, sensitivity $\left(d^{\prime}\right)$ and response criterion $(\ln \beta)$, of CPT performance were derived from hits (probability of response to target trials) and false alarms (probability of response to nontarget trials) (Nuechterlein, 1991). Variables extraneous to the intended comparison $\left(d^{\prime}\right)$, such as differences in motivation or in cooperativeness between the groups would yield differences in $\ln \beta$ rather than in $d^{\prime}$.

\subsection{Procedures}

After giving written informed consent for participation, all patients included in the MPGRP received standardized evaluations for baseline information, covering premorbid personality development, psychosocial history, clinical course of the disorder prior to the index admission and presenting symptoms at admission. Psychopathology was further evaluated by a semistructured interview using the PANSS-CH within 1 week after admission by attending psychiatrists who had completed the PANSS-CH reliability training. Sustained attention was assessed by the CPT when the patients became stable enough to complete the 15-min test. The PANSS-CH ratings were done with the evaluators blind to the results of CPT.

\subsection{Statistical analysis}

A principal component analysis was performed on the 14 PANSS-CH Positive and Negative scale items to explore the underlying factor structures. Not all 30 PANSS-CH items were included, because the subject/variable ratio would have been too low to result in stable factor structures (Andreasen et al., 1995). The criterion used to select the number of factors was eigenvalues greater than unity. The initial factor solution was then rotated to obtain simple structure, by using the quartimax procedure. Each case's ratings of individual PANSS items were multiplied by their corresponding loadings on the rotated factors, and the products were summed to yield empirical factor scores for each factor. The resulting empirical factor scores could be viewed as representing the severity of the corresponding symptom dimensions. For comparison, summed scores from the Positive and Negative scale items, respectively, 
were taken as a measure of overall severity of each syndrome (Kay, 1991).

Pearson product-moment correlation coefficients were calculated in assessing the relationship between two continuous variables, such as the summed scores of positive and negative syndromes, the empirical factor scores, $d^{\prime}$ and $\ln \beta$. Paired $t$ tests were used in comparing CPT performance indices for the undegraded and the degraded sessions. The relationship between individual factors of schizophrenic symptoms and CPT performance was further assessed by regressing $d^{\prime}$ or $\ln \beta$ on all empirical factor scores with age, sex and education as covariates. The regression coefficient of an individual empirical factor in this model thus reflects the amount of change in the $\mathrm{CPT}$ index, correlated with a unit change in this factor score, with the other factors and all demographic features held constant. For comparison, two other definitions of clinical symptoms were used in the multiple linear regression analyses: individual symptom (individual item ratings) and syndrome (the summed scores of the Positive and Negative scales). A $p$ value less than 0.05 was considered significant, while a $p$ value in the range of $0.05-0.1$ was considered borderline. All analyses were performed using the Statistical Package for Social Sciences (Norusis, 1986).

\section{Results}

Among the 60 schizophrenic patients, 28 (47\%) were male and $32(53 \%)$ were female. Because males and females were not different in demographic, clinical and neuropsychological characteristics, these features are presented for the whole sample only (Table 1). All of the patients were in acute episodes with moderate severity of symptoms, as revealed by the distributions of their scorings on Positive and Negative scale items. They had been receiving antipsychotic medications with or without concomitant benzodiazepine, $\beta$-blocker or anticholinergics, depending on clinical conditions of the patients.

Compared to the population mean of 345 community subjects (Chen et al., 1997a), i.e., 4.0 for undegraded $d^{\prime}$ and 3.4 for degraded $d^{\prime}$, the CPT
Table 1

Demographic, clinical and neuropsychological characteristics of 60 schizophrenic patients

\begin{tabular}{lcc}
\hline Variables & Mean & SD \\
\hline Age (years) & 28.2 & 7.7 \\
Education (years) & 10.8 & 3.2 \\
Age at onset (years) & 23.6 & 6.8 \\
Duration of illness (years) & 2.7 & 2.9 \\
PANSS ${ }^{\mathrm{a}}$ rating & & \\
Positive scale (summed score) & 19.3 & 7.1 \\
Negative scale (summed score) & 20.3 & 15.9 \\
Undegraded CPT ${ }^{\mathrm{b}}$ performance & & \\
$d^{\prime}$ & 1.7 & 1.5 \\
$\ln \beta$ & 0.2 & 0.2 \\
$25 \%$ degraded CPT performance & & \\
$d^{\prime}$ & 0.5 & 1.0 \\
$\ln \beta$ & 0.1 & 0.1 \\
\hline
\end{tabular}

${ }^{2}$ Positive and Negative Syndrome Scale (Kay et al., 1987).

${ }^{\mathrm{b}}$ Continuous Performance Test; $d^{\prime}=$ sensitivity and $\ln \beta=$ response criterion on the $\mathrm{CPT}$.

sensitivity of these patients on both degraded and degraded sessions was severely impaired (Table 1). The decrement in $d^{\prime}$ from the undegraded to the degraded session was significant (paired $t=7.31$, $\mathrm{df}=59, p<0.001$ ). Similarly, the response criteria of the patients were less stringent than those of a community population (Chen et al., 1997a), i.e., 2.2 for both the undegraded and the degraded CPT. The decrement in $\ln \beta$ from the undegraded to the degraded session was significant $(t=8.71$, $\mathrm{df}=59, p<0.001)$. The correlation between $d^{\prime}$ and $\ln \beta$ was not significant for either the undegraded $(r=0.13, p=0.30)$ or the degraded $(r=-0.16, p=$ $0.22) \mathrm{CPT}$.

The result of principal component analysis followed by quartimax rotation procedure of the 14 items yielded a four-factor solution ( Table 2). Five out of the seven PANSS Negative scale items loaded highly on the first factor. Since all the traditionally-defined negative symptoms were included (blunted affect, emotional withdrawal, poor rapport, passive social withdrawal and poverty of spontaneous speech), we defined it as the negative factor. The second factor consisted of four Positive scale items indicating reality distortion (delusion, hallucination, grandiosity and suspiciousness). This was termed as the delusion/hallucination factor. The other two 
Table 2

Factor loadings from a quartimax factor analysis of the 14 PANSS $^{\mathrm{a}}$ Positive and Negative scale items $(n=60)$

\begin{tabular}{|c|c|c|c|c|}
\hline Items & Negative & Delusion/hallucination & Disorganization & Excitement \\
\hline \multicolumn{5}{|l|}{ Positive scale } \\
\hline Delusion & 0.02 & $0.87^{b}$ & 0.06 & 0.17 \\
\hline Conceptual disorganization & 0.30 & 0.28 & 0.73 & 0.14 \\
\hline Hallucination & 0.36 & 0.78 & 0.05 & 0.06 \\
\hline Excitement & 0.23 & 0.24 & 0.19 & 0.78 \\
\hline Grandiosity & -0.39 & 0.49 & 0.34 & -0.25 \\
\hline Supiciousness & 0.32 & 0.71 & -0.03 & 0.36 \\
\hline Hostility & 0.38 & 0.24 & 0.09 & 0.75 \\
\hline \multicolumn{5}{|l|}{ Negative scale } \\
\hline Blunted affect & 0.78 & 0.05 & 0.27 & -0.18 \\
\hline Emotional withdrawal & 0.84 & 0.26 & 0.04 & 0.11 \\
\hline Poor rapport & 0.79 & 0.13 & 0.23 & 0.31 \\
\hline Passive/apathetic social withdrawal & 0.89 & 0.16 & -0.05 & 0.13 \\
\hline Poor abstract thinking & 0.30 & -0.01 & 0.75 & -0.11 \\
\hline Lack of spontaneity/flow of conversation & 0.64 & -0.26 & 0.34 & 0.10 \\
\hline Stereotyped thinking & 0.12 & -0.01 & 0.80 & 0.22 \\
\hline Variance explained $(\%)$ & 38.50 & 15.10 & 11.80 & 7.40 \\
\hline
\end{tabular}

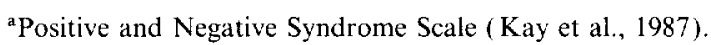

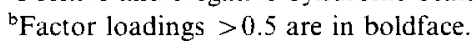

PANSS-CH negative symptoms (poor abstract thinking and stereotyped thinking) clustered with 'conceptual disorganization' of the Positive scale to form the third factor, which reflected disturbance of thought processes and was named the disorganization factor. The fourth factor was termed the excitement factor, since it consisted of 'excitement' and 'hostility' of the Positive scale and indicated the presence of psychomotor agitation. The four factors explained $72.8 \%$ of all variance.

For the 14 individual item ratings (seven for the Positive scale and seven for the Negative scale), two summed scores of the subscales and the four empirical factors, only 'blunted affect' had a borderline negative correlation with $d^{\prime}$ on the undegraded $(r=-0.22, p=0.07)$ and a significant negative correlation with $d^{\prime}$ on the degraded $(r=$ $-0.25, p=0.04) \mathrm{CPT}$. In contrast, 'delusion' $(r=$ $0.28, p=0.03)$, 'hallucination' $(r=0.26, p=0.04)$, 'hostility' $(r=0.26, p=0.04)$ and the summed score of the Positive scale $(r=0.30, p=0.02)$ showed significant correlation with the response criterion index, $\ln \beta$, on the undegraded CPT.

When CPT performance indices were regressed on the individual item ratings with sex, age and education as covariates, no association was detected. When the same procedures were repeated on the summed scores of Positive and Negative scales, only the summed score of the Negative scale had a borderline association with the $d^{\prime}$ on the undegraded CPT (regression coefficient $=$ $-0.29, p=0.08$ ). If the CPT performance indices were instead regressed on the four empirical factors with sex, education and age as covariates, both the negative and the excitement factors had significant associations with the $d^{\prime}$ in both the undegraded and the degraded sessions (Table 3 ). The negative factor predicted poorer sensitivity, while the excitement factor predicted greater sensitivity.

\section{Discussion}

The results of the factor analysis clearly showed that the clinical symptoms included in the Positive or Negative scales of PANSS could be further divided into at least four distinct symptom dimensions: negative, disorganization, delusion/hallucination and excitement. Previous factor analytic studies of the PANSS using all three scales have indicated a five-factor structure that consists of 
Table 3

Coefficients for the regression analysis of the $\mathrm{CPT}^{\mathrm{a}}$ performance index on the four empirical factors with age, education and sex as covariates

\begin{tabular}{|c|c|c|c|c|}
\hline \multirow[t]{2}{*}{ CPT index } & \multicolumn{4}{|c|}{ Regression coefficient $(\mathrm{SE}) \times 100$} \\
\hline & Negative & Delusion/hallucination & Disorganization & Excitement \\
\hline \multicolumn{5}{|l|}{ Undegraded } \\
\hline$d^{\prime}$ & $-0.16(0.06)^{*}$ & $-0.07(0.07)$ & $-0.03(0.08)$ & $0.39(0.06)^{* *}$ \\
\hline $\ln \beta$ & $0.00(0.08)$ & $0.12(0.09)$ & $-0.05(0.11)$ & $0.05(0.18)$ \\
\hline \multicolumn{5}{|c|}{$25 \%$ degraded } \\
\hline$d^{\prime}$ & $-0.10(0.04)^{*}$ & $-0.05(0.05)$ & $0.01(0.05)$ & $0.25(0.04)^{* *}$ \\
\hline $\ln \beta$ & $0.08(0.07)$ & $0.03(0.08)$ & $0.04(0.09)$ & $-0.22(0.15)$ \\
\hline
\end{tabular}

${ }^{\text {a }}$ Continuous Performance Test; $d^{\prime}=$ sensitivity and $\ln \beta=$ response criterion on the CPT.

${ }^{*} p<0.005,{ }^{* *} p<0.01$ for the $z$-test.

negative, positive, cognitive, excitement and depressive factors (Kay and Sevy, 1990; Lindenmayer et al., 1995). The depressive factor mainly contains symptoms included in the General Psychopathology scale, which were not used in this study. Otherwise, the structure and compositions of the remaining four factors obtained from these studies showed striking similarities to ours.

The separation of the items in the PANSS Negative scale into two distinct symptom dimensions (negative and disorganization) was consistent with the results of recent large-scale exploratory and confirmatory factor analytic studies using different scales (Lindenmayer et al., 1995; Johnstone and Frith, 1996; Lenzenweger and Dworkin, 1996; Vázquez-Barquero et al., 1996). Meanwhile, as previous studies have indicated (Klimidis et al., 1993; Miller et al., 1993), the composition of positive syndrome tends to be more heterogeneous than that of negative syndrome. The items in the Positive scale separated into the delusion/hallucination factor, excitement factor and part of the disorganization factor. Among them, the delusion/hallucination factor has also been consistently extracted in studies using the global ratings of the Scale for the Assessment of Positive Symptoms subscales (Andreasen and Olsen, 1982; Liddle, 1987; Klimidis et al., 1993; Miller et al., 1993). However, when individual ratings of various types of hallucinations and delusions were used in factor analysis, a paranoid/non-paranoid subdivision of the hallucination/delusion factor was discerned (Silver et al., 1993; Vázquez-Barquero et al., 1996).

Further support for the construct validity of these four factors comes from their relationship with CPT performance. Although none of the four empirical factors correlated with $d^{\prime}$ univariately, the associations between negative as well as excitement factors and $d^{\prime}$ became significant after age, sex, education and the other symptom factors were controlled for. In contrast, the association between 'blunted affect' and $d$ ' became nonsignificant after demographic features and other items were controlled for. These results highlight the importance of adjustment for demographic features and other coexisting symptoms when comparing the CPT performance of schizophrenic patients. In the acute phase of schizophrenia, patients tend to have multiple symptoms (Carpenter et al., 1991). Since the empirical factors are orthogonally derived from the results of principal component analysis, the effect of individual factors are thus more readily discernible than individual item or summed scores. In our study, the results of multiple regression analyses showed that symptom dimensions derived from empirical factor analysis are more accurate than individual symptoms or global severity of syndrome in predicting $d^{\prime}$ of the CPT.

Previous studies have also found that the summed scores of the hypothetical Anergia factor from the Brief Psychiatric Rating Schedule (BPRS) (Nuechterlein et al., 1986) and the summed scores of the Scale for the Assessment of 
Negative Symptoms global item ratings (representing the severity of the negative syndrome) (Hain et al., 1993) were associated with lower $d^{\prime}$ on the CPT. However, other hypothetical BPRS negative factors (emotional withdrawal, motor retardation, and blunted affect) have no association with $d^{\prime}$ on the CPT (Strauss et al., 1993). The failure to use empirical factors and make adjustments for demographic features and other symptom dimensions might account for the discrepancies.

Studies among nonclinical populations have also demonstrated that the negative factor or interpersonal dysfunction of schizotypal personality features were associated with lower $d^{\prime}$ on the CPT (Kendler et al., 1991; Chen et al., 1997b). In addition, the severity of negative symptoms among the unaffected first-degree relatives of schizophrenic probands has been shown to be higher than that of the relatives of affective disorder probands (Tsuang et al., 1991). Taken together with the finding that negative symptoms seem to predict a higher concordance rate in schizophrenic siblings (McGuffin et al., 1991), it appears that negative symptoms may carry a higher genetic loading of schizophrenia.

This raises an important issue: are negative schizophrenic symptoms an expression of a stable underlying trait rather than of an episodic exacerbation? A few longitudinal factor analytic studies have shown that, although symptoms of schizophrenia fluctuated over time, the underlying structure of symptoms remained rather stable (Arndt et al., 1995; Lindenmayer et al., 1995). Among the symptom structures reported, the negative symptom dimension was consistently included. In non-schizophrenic populations, although the factor structure of schizotypal symptoms did not entirely correspond to the factor structure of schizophrenia symptoms, factors representing negative symptoms were clearly discernible (Raine et al., 1994; Vollema and van den Bosch, 1995; Bergman et al., 1996; Chen et al., 1997b). Thus, despite the fluctuation in severity, the negative symptom dimension may constitute a stable component in the latent symptom structure of schizophrenia and schizophrenic spectrum disorders.

The association between the negative dimension and lower $d^{\prime}$ on the CPT indicates that certain clinical symptoms of schizophrenia are the result of a breaking down of the proper gating and processing of incoming information (ErlenmeyerKimling et al., 1993). Attention impairment is especially critical in leading to a subject's inability to process complex interpersonal cues and communications, and hence to a difficulty in interpersonal interaction.

The association between the excitement symptom dimension and higher $d^{\prime}$ on both the undegraded and the degraded CPT was unexpected. This positive correlation indicates that schizophrenic patients with a higher level of excitement did better than those with other symptom profiles, although all of them scored well below the population mean (Chen et al., 1997a). Since this factor was less extracted from previous factor-analytic studies of schizophrenic symptoms, it is difficult to examine the consistency of the findings across studies. This question warrants further exploration.

In contrast, the delusion/hallucination dimension does not correlate with either $d^{\prime}$ or $\ln \beta$ on the CPT. Only individual items of delusion and hallucination and the summed score of the Positive scale had a positive correlation with $\ln \beta$ on the undegraded CPT. However, the correlation disappeared after the demographic variables were adjusted for. These results are consistent with previous reports that florid psychotic symptoms influenced only the response criterion and not the $d^{\prime}$ on the CPT (Cornblatt et al., 1985).

The lack of association between the disorganization dimension and CPT performance indices in this study might have been due to the limited number of items included for assessing formal thought disorders in the PANSS. When a more comprehensive instrument for thought disorders was used, a correlation between formal thought disorders and lower $d^{\prime}$ of the CPT was demonstrated (Nuechterlein et al., 1986; Pandurangi et al., 1994). This indicates that in future studies of clinical symptoms and CPT performance indices, the PANSS should incorporate more items for addressing formal thought problems, or a supplementary scale specifically for formal thought disorders should be employed. 
Although results of the present study provide further insight into the clinical symptoms of schizophrenia, sustained attentional deficits and their relationships, some problems should be borne in mind when interpreting the results. In the acute stage, patients often show myriad fleeting symptoms that might be phase-specific and of diverse origin, which are prone to change as medical intervention is initiated. Although symptom dimensions have been demonstrated to be stable during both acute stage (Lindenmayer et al., 1995) and long-term course (Arndt et al., 1995), whether their relationships with the sustained attentional deficits are stable throughout the course of the illness warrants further investigations. Furthermore, the mechanism underlying the relationships between these symptom dimensions and CPT performance deficits were not explored in our study. Future studies on the action of neurotransmitter systems on cognitive functions and neuropsychological performance will be valuable in this regard.

\section{Acknowledgment}

This work was supported by a research grant from National Health Research Institute, Taiwan (DOH83-HR-306, DOH84-HR-306 and DOH85HR-306). The research team members of the Multidimensional Psychopathology Group Research Projects (MPGRP), including Drs. Ching-Jui Chang, Hung-Jung Chang, Hai Ho, Ping-Ju Chang, Shi-Chin Guo, Shian-Yuan Lan, Su-Kuan Lin, Fu-Chuan Wei and Joseph J. Cheng, performed the clinical interviews. We thank LinLin Yeh for her work in coordination of the MPGRP and for her help in the statistical analysis, and Shan-Ying Liu for the preparation of the manuscript.

\section{References}

American Psychiatric Association, 1987. Diagnostic and Statistical Manual of Mental Disorders (Third Edition, Revised). American Psychiatric Association, Washington, DC.

Andreasen, N.C., Olsen, S., 1982. Negative vs positive schizo- phrenia: definition and validation. Arch. Gen. Psychiatry $39,789-794$

Andreasen, N.C., Arndt, S., Alliger, R., Miller, D., Flaum, M., 1995. Symptoms of schizophrenia: methods, meanings, and mechanisms. Arch. Gen. Psychiatry 52, 341-351.

Arndt, S., Alliger, R.J., Andreasen, N., 1991. The distinction of positive and negative symptoms: the failure of a twodimensional model. Br. J. Psychiatry 158, 317-322.

Arndt, S., Andreasen, N.C., Flaum, M., Miller, D., Nopoulos, P., 1995. A longitudinal study of symptom dimensions in schizophrenia: prediction and pattern of change. Arch. Gen. Psychiatry 52, 352-359.

Asarnow, R.F., MacCrimmon, D.J., 1978. Residual performance deficit in clinically remitted schizophrenics: a marker of schizophrenia? J. Abnorm. Psychol. 87, 597-608.

Bergman, A.J., Harvey, P.D., Mitropoulou, V., Aronson, A., Marder, D., Silverman, J., Siever, L.J., 1996. The factor structure for schizotypal symptoms in a clinical population. Schizophr. Bull. 22, 501-509.

Berrios, G.E., 1991. Positive and negative signals: a conceptual history. In: Marneros, A., Andreasen, N.C., Tsuang, M.T. (Eds.), Negative versus Positive Schizophrenia. SpringerVerlag, Berlin, pp. 8-27.

Carpenter, W.T., Buchana, R.W., Kirkpatrick, B., Thaker, G., Tamminga, C., 1991. Negative symptoms: a critique of current approaches. In: Marneros, A., Andreasen, N.C., Tsuang, M.T. (Eds.), Negative versus Positive Schizophrenia. Springer-Verlag, Berlin, pp. 126-133.

Chen, W.J., Hsiao, C.K., Hsiao, L.L., Hwu, H.G., 1997a. Performance of the Continuous Performance Test among community samples. Schizophr. Bull. (in press).

Chen, W.J., Hsiao, C.K., Lin, C.C.H., 1997b. Schizotypy in community samples: the three-factor structure and correlation with sustained attention. J. Abnorm. Psychol. (in press).

Cheng, J.J., Ho, H., Chang, C.J., Lan, S.Y., Hwu, H.G., 1996. Positive and Negative Syndrome Scale (PANSS): establishment and reliability study of a Mandarin Chinese language version. Chinese Psychiatry 10, 251-258.

Cornblatt, B.A., Lenzenweger, M.F., Dworkin, R.H., Erlenmeyer-Kimling, L., 1985. Positive and negative schizophrenic symptoms, attention, and information processing. Schizophr. Bull. 11, 397-408.

Cornblatt, B.A., Lenzenweger, M.F., Erlenmeyer-Kimling, L. 1989. The Continuous Performance Test, Identical Pairs version: II. Contrasting attentional profiles in schizophrenic and depressed patients. Psychiatry Res. 29, 65-86.

Cornblatt, B.A., Lenzenweger, M.F., Dworkin, R.H., Erlenmeyer-Kimling, L., 1992. Childhood attentional dysfunctions predict social deficits in unaffected adults at risk of schizophrenia. Br. J. Psychiatry 161 (suppl. 8), 59-64.

Crow, T.J., 1980. Molecular pathology of schizophrenia: more than one disease process? Br. Med. J. 280, 66-68.

De Leon, J., Simpson, G.M., Peralta, V., 1992. Positive and negative symptoms in schizophrenia: where are the data? Biol. Psychiatry $31,431-434$.

Erlenmeyer-Kimling, L., Cornblatt, B.A., Rock, D., Roberts, S., Bell, M., West, A., 1993. The New York High-Risk 
Project: anhedonia, attentional deviance, and psychopathology. Schizophr. Bull. 19, 141-153.

Hain, H., Maier, W., Klinger, T., Franke, P., 1993. Positive/negative symptomatology and experimental measures of attention in schizophrenia. Psychopathology 26, $62-68$.

Johnstone, E.C., Frith, C.D., 1996. Validation of three dimensions of schizophrenic symptoms in a large unselected sample of patients. Psychol. Med. 26, 669-679.

Kay, S.R., 1991. Positive and Negative Syndromes in Schizophrenia: Assessment and Research. Brunner/Mazel, New York.

Kay, S.R., Sevy, S., 1990. Pyramidal model of schizophrenia. Schizophr. Bull. 16, 537-545.

Kay, S.R., Fiszbein, A., Opler, L.A., 1987. The Positive and Negative Syndrome Scale (PANSS) for schizophrenia. Schizophr. Bull. 13, 261-276.

Kendler, K.S., Ochs, A.L., Gorman, A.M., Hewitt, J.K., Ross, D.E., Mirsky, A.F., 1991. The structure of schizotypy: a pilot multitrait twin study. Psychiatry Res. 36, 19-36.

Klimidis, S., Stuart, G.W., Minas, I.H., Copolov, D.L., 1993. Positive and negative symptoms in the psychoses: reanalysis of published SAPS and SANS global ratings. Schizophr. Res. 9, 11-18.

Lenzenweger, M.F., Dworkin, R.H., 1996. The dimensions of schizophrenia phenomenology: not one or two, at least three, perhaps four. Br. J. Psychiatry $168,432-440$.

Liddle, P.F., 1987. The symptoms of chronic schizophrenia: a reexamination of the positive-negative dichotomy. $\mathrm{Br}$. J. Psychiatry 151, 145-151.

Lindenmayer, J.P., Grochowski, S., Hyman, R.B., 1995. Five factor model of schizophrenia: replication across samples. Schizophr. Res. 14, 229-234.

McGuffin, P., Harvey, I., Williams, M., 1991. The positive/negative dichotomy: does it make sense from the perspective of the genetic researcher? In: Marneros, A., Andreasen, N.C., Tsuang, M.T. (Eds.), Negative versus Positive Schizophrenia. Springer-Verlag, Berlin, pp. 250-264.

Miller, D.D., Arndt, S., Andreasen, N.C., 1993. Alogia, attentional impairment, and inappropriate affect: their status in the dimension of schizophrenia. Comp. Psychiatry 34, 221-226.

Mussgay, L., Hertwig, R., 1990. Signal detection indices in schizophrenics on a visual, auditory and bimodal Continuous Performance Test. Schizophr. Res. 3, 303-310.

Neale, J., Oltmanns, T., Harvey, P., 1985. The need to relate cognitive deficits to specific behavior referents of schizophrenia. Schizophr. Bull. 11, 286-291.
Norusis, M.J., 1986. The Statistical Package for Social Sciences (SPSS), Manual of Reference. SPSS, Inc., Chicago.

Nuechterlein, K.H., 1991. Vigilance in schizophrenia and related disorders. In: Nasrallah, H.A. (Series Ed.), Handbook of Schizophrenia, Vol. 5, Steinhauer, S.R., Gruzelier, J.H., Zubin, J. (Eds.), Neuropsychology, Psychophysiology and Information Processing. Elsevier, Amsterdam, pp. 397-433.

Nuechterlein, K.H., Edell, w.S., Norris, M., Dawson, M.E., 1986. Attentional vulnerability indicators, thought disorder, and negative symptoms. Schizophr. Bull. 12, 408-426.

Orzack, M.H., Kornetsky, C., 1966. Attention dysfunction in chronic schizophrenia. Arch. Gen. Psychiatry 14, 323-327.

Pandurangi, A.K., Sax, K.W., Pelonero, A.L., Goldberg, S.C., 1994. Sustained attention and positive formal thought disorder in schizophrenia. Schizophr. Res. 13, 109-116.

Raine, A., Reynolds, C., Lencz, T., Scerbo, A., Triphon, N., Kim, D., 1994. Cognitive-perceptual, interpersonal, and disorganized features of schizotypal personality. Schizophr. Bull. 20 (1), 191-201.

Rosvold, H.E., Mirsky, A.F., Sarason, I., Bransome, E.D., , Jr.Bech, L.H., 1956. A Continuous Performance Test of brain damage. J. Consult. Psychol. 20, 343-350.

Rutschmann, J., Cornblatt, B., Erlenmeyer-Kimling, L., 1977. Sustained attention in children at high risk for schizophrenia: report on a Continuous Performance Test. Arch. Gen. Psychiatry 34, 571-575.

Silver, H., David, D., Kaplan, M., Hadjez, J., 1993. Factor analysis of schizophrenic symptoms and comparison of different rating scales. Schizophr. Res. 10, 67-75.

Strauss, M.E., Buchanan, R.W., Hale, J., 1993. Relationships between attentional deficits and clinical symptoms in schizophrenic outpatients. Psychiatry Res. 47, 205-213.

Tsuang, M.T., Gilbertson, M.W., Faraone, S.V., 1991. Genetic transmission of negative and positive symptoms in the biological relatives of schizophrenics. In: Marneros, A., Andreasen, N.C., Tsuang, M.T. (Eds.), Negative versus Positive Schizophrenia. Springer-Verlag, Berlin, pp. 265-291.

Vázquez-Barquero, J.L., Lastra, I., Nuñez, M.J.C., Castanedo, S.H., Dunn, G., 1996. Patterns of positive and negative symptoms in first episode schizophrenia. Br. J. Psychiatry 168 , 693-701.

Vollema, M.G., van den Bosch, R.J., 1995. The multidimensionality of schizotypy. Schizophr. Bull. 21, 19-31.

Wohlberg, G.W., Kornetsky, C., 1973. Sustained attention in remitted schizophrenics. Arch. Gen. Psychiatry 28, 533-537. 